\title{
Spiking network modeling of neuronal dynamics in individual rats
}

\author{
John S Choi ${ }^{1}$, Rosemary J Menzies ${ }^{2}$, Salvador Dura-Bernal ${ }^{1}$, Joseph T Francis ${ }^{1}$, William W Lytton ${ }^{1}$, Cliff C Kerr ${ }^{1,2,3^{*}}$ \\ From 24th Annual Computational Neuroscience Meeting: CNS*2015 \\ Prague, Czech Republic. 18-23 July 2015
}

Many researchers base their neuronal models on experimental data, but few systematically calibrate to it, and even fewer use unpooled data from multiple subjects. Some (partial) exceptions are [1], where one model parameter was calibrated to data pooled across four macaques; and [2], where five model parameters were calibrated to unpooled data from 292 humans. To our knowledge, no one has previously calibrated spiking network models to individual subjects - likely because a single model iteration typically takes considerable computational time, compounded by the formidable number of iterations required to perform optimizations on a statistically meaningful number of subjects.

In this study, we calibrate and validate a mesoscopic spiking network model against data from individual rats, then use these fits to infer differences in the rats' physiologies. We recorded data from microelectrode arrays implanted in the somatosensory cortices of nine male rats, each of whom received touch stimuli to his left forepaw. The spiking network models consisted of 20,000 Izhikevich neurons, representing a $2 \times 2 \mathrm{~mm}$ patch of cortex sampled at approximately $10 \%$ true cell density, with cell types (15 across six layers) and connectivities based on empirical data. We calibrated key model parameters (including connection probability and weight, tonic background activity, and the ratio of thalamic to cortical input) to experimental data (including average firing rates and coefficients of variation) using a nonlinear optimization algorithm [3]. The calibrations were validated using the exponents of the local field potential (LFP) power spectra from 5-50 Hz, which were not used for calibration. Changes in the information processing

\footnotetext{
* Correspondence: cliff@thekerrlab.com

${ }^{1}$ Department of Physiology and Pharmacology, SUNY Downstate Medical Center, Brooklyn, NY 10029, USA

Full list of author information is available at the end of the article
}

properties of the (simulated) networks were then quantified via interlaminar Granger causality.

Experimentally, we found large differences between subjects. Cortical firing rates varied from $3.8 \mathrm{~Hz}$ to 19.6 $\mathrm{Hz}$ (median $11.9 \mathrm{~Hz}$ ); coefficients of variation, 0.4 to 1.2 (median 0.9); and peristimulus time histogram peak amplitudes, $48 \mathrm{~Hz}$ to $73 \mathrm{~Hz}$ (median $62 \mathrm{~Hz}$ ). Inter-subject differences were significantly greater than intra-subject differences across both sessions and electrodes. The calibrated models reproduced experimental data with an average mismatch of $16 \%$, compared to $30 \%$ when uncalibrated. Experimentally, the LFP power spectra exponents varied from -1.4 to -2.7 (median -2.2). While the models had uniformly steeper exponents (range -2.8 to -3.6), calibration reduced mismatch in eight of the nine subjects. Inter-subject differences could be largely accounted for by differences in average synaptic connection probability (which varied by a mean of $24 \%$ between subjects, compared to $<8 \%$ for other fitted parameters). Parameter differences produced changes in how these networks process information - for example, there were significant and complex differences in the patterns of interlaminar information flow between subjects, including reversals in the dominant direction of information flow for some layer pairs.

In summary, we found that (1) significant differences exist between individual subjects, (2) these differences can be captured by calibrating spiking network models to data from each individual, and (3) modeling suggests that these differences affect how individuals process information across cortical layers.

\footnotetext{
Authors' details

'Department of Physiology and Pharmacology, SUNY Downstate Medical Center, Brooklyn, NY 10029, USA. ${ }^{2}$ Complex Systems Group, School of Physics, University of Sydney, Sydney, NSW 2006, Australia. ${ }^{3}$ Centre of Excellence for Integrative Brain Function, University of Sydney, Sydney, NSW 2006, Australia
} 
Published: 18 December 2015

\section{References}

1. Rasch MJ, Schuch K, Logothetis NK, Maass W: Statistical comparison of spike responses to natural stimuli in monkey area $\mathrm{V} 1$ with simulated responses of a detailed laminar network model for a patch of $\mathrm{V} 1$. J Neurophysiol 2011, 105(2):757-78.

2. Kerr CC, Kemp AH, Rennie CJ, Robinson PA: Thalamocortical changes in major depression probed by deconvolution and physiology-based modeling. Neuroimage 2011, 54(4):2672-82.

3. Kerr CC, Smolinski TG, Dura-Bernal S, Wilson DP: Optimization by Bayesian adaptive locally linear stochastic descent, Under review; available at http:// thekerrlab.com/pubs/ballsd.pdf.

\section{doi:10.1186/1471-2202-16-S1-P122}

Cite this article as: Choi et al: Spiking network modeling of neuronal dynamics in individual rats. BMC Neuroscience 2015 16(Suppl 1):P122.

\section{Submit your next manuscript to BioMed Central} and take full advantage of:

- Convenient online submission

- Thorough peer review

- No space constraints or color figure charges

- Immediate publication on acceptance

- Inclusion in PubMed, CAS, Scopus and Google Scholar

- Research which is freely available for redistribution

Submit your manuscript at www.biomedcentral.com/submit
C Biomed Central 